EPJ manuscript No.

(will be inserted by the editor)

\title{
Excitations of anisotropic spin-1 chains with matrix product ground state
}

\author{
E. Bartel, A. Schadschneider, and J. Zittartz \\ Institut für Theoretische Physik, Universität zu Köln \\ Zülpicher Straße 77, D-50937 Köln, Germany \\ Received: date / Revised version: date
}

\begin{abstract}
We investigate a large class of antiferromagnetic spin-1 chains with nearest neighbour interaction and exactly known matrix product ground state. The spectrum of low-lying excitations is calculated numerically by DMRG and exact diagonalisation. Spin liquid behaviour with an excitation gap is observed meeting the Haldane scenario. Further, we compare properties of the anisotropic model with the wellknown isotropic AKLT model and use the analytical single mode approximation to obtain a quantitative understanding of the excitation gap. The low-lying excited states can be interpreted in terms of a magnonlike elementary excitation.
\end{abstract}

PACS. 75.10.Jm Quantized spin models.

\section{Introduction}

Quantum spin systems have been studied intensively in recent years (for a review, see e.g. 1]). Special interest has been taken in the case of low dimensions where strong quantum fluctuations destroy magnetic order [2]. Antiferromagnetic spin chains with $S=1$ have been the focus of theoretical and experimental studies since Haldane's conjecture [3] about the difference between integer and halfinteger spins. Antiferromagnetic integer spin chains are predicted to be generically massive while half-integer spin ' chains have a vanishing gap which can be rigorously shown for the isotropic spin- $\frac{1}{2}$ chain using the Bethe ansatz so' lution 4. For the isotropic Heisenberg $S=1$ chain with nearest neighbour exchange no exact solution has been found, but there is convincing evidence from numerical ' studies that a finite gap exists in the thermodynamic limit [5].

In a most general spin-1 Hamiltonian, bilinear as well as biquadratic interaction terms along with possible anisotropies emerge. Consideration of only bilinear exchange with anisotropic interaction and single-ion anisotropies leads to a well-known two-parameter phase diagram which includes massive as well as massless phases (see e.g. [6] and references therein). The spin-1 Hamiltonian with isotropic nearest neighbour - bilinear and biquadratic - interaction has also been focus to extensive theoretical studies, see e.g. 7, $8,9,10,11,12,13,14,15$. This one-parameter model including the Heisenberg Hamiltonian exhibits a variety of magnetic phenomena like ferromagnetism, dimerization and a gapped spin liquid (Haldane) phase. A valencebond-solid model (AKLT model) with exactly known ground state representing this phase has been introduced by Af- fleck et al. [16. Only a few isotropic models with antiferromagnetic bilinear and biquadratic exchange possess exactly known spectra [17,18,19,20,21. While experiments on many spin-1 compounds $22,23,24,25,26$ suggest that biquadratic exchange can usually assumed to be small, Mila and Zhang [27] presented a microscopic model with non-negligible biquadratic exchange in order to understand a massless phase of the spin- 1 vanadium oxide $\mathrm{LiVGe}_{2} \mathrm{O}_{6}$.

In 28] a large class of spin-1 models possessing a unique matrix product ground state was introduced. This model class includes biquadratic terms and anisotropic exchange at the same time and has been proven rigorously to exhibit exponentially decaying spin-spin-correlations. In the isotropic limit it reduces to the AKLT model [16. While the ground state and some of its properties have been calculated exactly the excitation spectrum in the anisotropic case has not been subject of studies yet. It has only been known that generically a finite gap is observed, leading to spin-liquid behaviour.

In this work we present numerical and analytical evidence for a finite excitation gap and discuss the spectrum and structure of low-lying excitations. Results from an analytical single mode approximation capture the nature of the lowest excited states and give an exact upper bound to the excitation gap.

\section{Model and symmetries}

We consider a spin-1 chain with rotational invariance in the $(x, y)$-plane, invariance under $S^{z} \rightarrow-S^{z}$ and translation and parity invariance, i.e. invariance under the exchange $j \leftrightarrow j+1$. The most general Hamiltonian with 
nearest neighbour interactions satisfying the above symmetries

$$
\begin{aligned}
H=\sum_{j} & \left\{J_{1}\left(S_{j}^{x} S_{j+1}^{x}+S_{j}^{y} S_{j+1}^{y}+\Delta S_{j}^{z} S_{j+1}^{z}\right)\right. \\
& +J_{2}\left(S_{j}^{x} S_{j+1}^{x}+S_{j}^{y} S_{j+1}^{y}\right)^{2}+J_{3}\left(S_{j}^{z} S_{j+1}^{z}\right)^{2} \\
& +J_{4}\left[\left(S_{j}^{x} S_{j+1}^{x}+S_{j}^{y} S_{j+1}^{y}\right)\left(S_{j}^{z} S_{j+1}^{z}\right)+\text { h.c. }\right] \\
& \left.+D\left(S_{j}^{z 2}+S_{j+1}^{z}{ }^{2}\right)+c\right\}
\end{aligned}
$$

gives rise to a model class with seven parameters including an energy off-set $c$ and a scale, reducing the number of relevant parameters to five. The additive constant $c$ is used to shift the ground state energy to $E_{0}=0$. The Hamiltonian (11) includes the bilinear, anisotropic spin-1 chain $\left(J_{2}=J_{3}=J_{4}=0\right)$ as well as a chain with only isotropic bilinear and biquadratic exchange $\left(\Delta=1, J_{2}=J_{3}=\right.$ $J_{4}, D=0$ ), both comprising the Heisenberg model.

A large subclass of the general model class (1) has been found 28 to possess a unique ground state which can be written as a product

$$
\left|\psi_{0}\right\rangle=\operatorname{tr}\left(m^{(1)} \cdot m^{(2)} \cdots m^{(L)}\right)
$$

of state matrices

$$
m^{(j)}=\left(\begin{array}{rr}
|0\rangle_{j} & \sqrt{a}|+\rangle_{j} \\
\sqrt{a}|-\rangle_{j} & \sigma|0\rangle_{j}
\end{array}\right), \quad a \neq 0, \quad \sigma \pm 1
$$

characterised by the real parameter ${ }^{1} a$ and the discrete parameter $\sigma= \pm 1$. Minimizing not only the total energy, but the energy of the local two-site Hamiltonian as well, the matrix product ground state (MPG) belongs to the class of optimum ground states [28. The parameter $a$ represents a degree of freedom connected to the anisotropy of the model. Further, taking excited states into account, three positive real spectral parameters $\lambda_{0}, \lambda_{1}, \lambda_{2}$ complete the parameter space of the MPG model. Neglecting a trival scale three relevant real parameters and one discrete parameter survive. For models with MPG a convenient representation of the spin-1 Hamiltonian makes use of spectral parameters and projection operators (see appendix) rather than spin operators (1) and shall be employed in this paper.

Referring to the spin-1 model class, among others, two unitary transformations

$$
\mathcal{D}^{1}=\prod_{j} e^{i \frac{\pi}{2} S_{j}^{z 2}}, \quad \mathcal{D}^{2}=\prod_{j \in A} e^{i \pi S_{j}^{z}}
$$

with $A$ denoting the subchain of even sites, mapping the parameter space onto itself can be found. Corresponding Hamiltonians show identical energy spectra, thus reducing the parameter space we need to discuss. The unitary transformation $\mathcal{D}^{1}$ maps the parameter $a$ on the model

\footnotetext{
1 Note that we choose the parameter $a$ in a different way compared to 28] in order to stress the spin-flip symmetry of the state matrix $m^{(j)}$.
}

with $-a$. It has no impact on the momentum. Therefore, in the following we concentrate on $|a|$. The second unitary transformation $\mathcal{D}^{2}$ changes model parameters $(a, \sigma)$ to $(-a,-\sigma)$. Please note that the momentum of odd- $S^{z}$ states is modified from $k$ to $\pi-k$ whereas the momentum of states with even $S^{z}$ remains unchanged under this transformation.

While the ground state is determined by only the anisotropy parameter $a$ and the discrete parameter $\sigma$, examination of the excitation spectrum requires consideration of the spectral parameters as well. Choosing the parameters ${ }^{2}$ $\lambda_{0}=\lambda_{1}=\lambda_{2}=1, a=-2, \sigma=-1$, an isotropic point of the MPG model class is reached. This special case corresponds to the AKLT model and has been studied intensively 162930 . Therefore, with fixed $\lambda_{0}=\lambda_{1}=\lambda_{2}=1$ we vary the parameter $|a|$.

\section{Numerical approach to low-lying excitations}

Low-lying excitations of the model are obtained by using density matrix renormalisation group (DMRG) 31,32 and exact diagonalisation data. Our DMRG-program implements the infinite system algorithm with conservation of the quantum number $S^{z}$ as well as periodic boundary conditions. Though usually a higher accuracy of DMRG data is achieved for open boundary conditions we have chosen periodic boundary conditions for a better comparison with analytical calculations for spin chains of different lengths. Moreover, dealing with a gapped system including a unique matrix product ground state the calculated energies are supposed to converge quickly 33. This has already been observed for the isotropic case in the vicinity of the AKLT point 11,12. In order to check the accuracy of our data we tested the convergence of the numerical method related to the chain length $L$ and to the number $m$ of states kept in each DMRG-iteration as well. With $L=100, m \leq 120$ reliable gap information for the MPG model class could be achieved. Since in real space DMRG momentum is not preserved we used exact diagonalisation of smaller chains $(L \leq 14)$ to discuss the momentum of the excitation levels.

\subsection{Isotropic interactions (AKLT model)}

Before we discuss the general case we briefly recall the results for the isotropic Hamiltonian $(a=-2, \sigma=-1)$. In fact the excitation spectrum of the AKLT model has been studied using numerical and analytical methods 12 , 34 and is well understood. The elementary excitations are magnon-type spin- 1 particles. The excitation gap is determined by the lowest energy $E_{S}(k)$ of the triplet excitations $(S=1)$ with momentum $k=\pi$ which belongs to a discrete branch below a continuum. The second excited state at $k=\pi$ has energy $3 E_{1}(\pi)$ and $\operatorname{spin} S=3$. On the other hand, the gap at momentum $k=0$ is twice as large as that at $k=\pi$ and has $\operatorname{spin} S=2$ [12. This suggests

${ }^{2}$ This equals $J_{1}=\frac{1}{2}, \Delta=1, D=0, J_{2}=J_{3}=J_{4}=\frac{1}{6}$. 


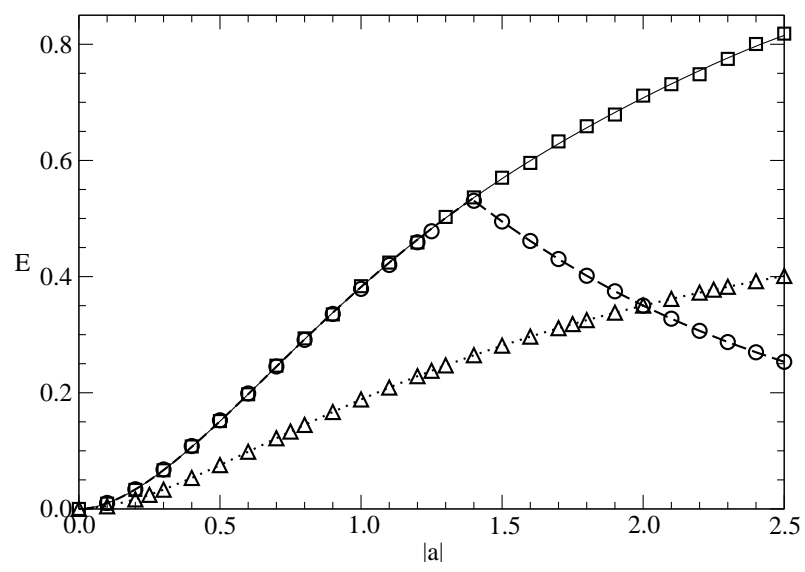

Fig. 1. Lowest excitations in the $S^{z}=0(\circ), 1(\triangle), 2$ ( $)$ subspaces plotted versus anisotropy parameter $|a|$ (DMRG-data).

that the corresponding states are scattering states of three resp. two magnons with $S=1$ and momentum $k=\pi$.

\subsection{Influence of anisotropy}

In fig. 1 the numerically calculated lowest excitations in three $S^{z}$-subspaces are plotted as function of the parameter $|a|$. For $|a| \neq 0$ a finite excitation gap to the unique ground state is observed [28]. At the point $a=0$ the model reaches a higher symmetry (invariance under transformation $\mathcal{D}^{1}$ ) leading to a three-fold degenerate ground state with vanishing excitation gap in the thermodynamic limit. At the isotropic AKLT model with $a=-2$ and $\sigma=-1$ the lowest excitation is a triplet with total spin $S=1$ and momentum $k=\pi$, in agreement with previous results (see Sec.3.11). A degeneracy of the energy levels in the $S^{z}=0,1$ subspaces can thus be seen at $|a|=2$. For smaller values of $|a|$ the anisotropy is of easy-plane type leading to a splitting of the lowest excitation level. Here, excitations along the z-axis are energetically favourable. Therefore the lowest excitations are found in the $S^{z}=1$ subspaces. In contrast, for $|a|>2$ an easy-axis anisotropy favours excitations with a vanishing total $S^{z}$. Further, we observe a level crossing in the $S^{z}=0$ subspace at $a=a_{l} \approx 1.4$. An obvious conjecture, consistent with the numerics, would be $a_{l}=\sqrt{2}$. At this point the interaction parameter $J_{3}$ changes sign. However, for a different choice of anisotropy parameters $\lambda_{j}$ the level crossing seems not to be related to such a change of sign. It can even be observed in cases where there is no sign change at all (see Sec. 3.4).

For smaller $|a|$ the lowest excitation with vanishing $S^{z}$ degenerates with the lowest energy levels in the $S^{z}=2$ subspaces. Its energy is close to double the size of the $S^{z}=$ 1 excitation gap. This gives rise to the conjecture that the lowest excitations in the $S^{z}=0,2$ subspaces consist of two $S^{z}=1$ excitations. In the following sections we find more evidence for this picture. For $a>a_{l}$ a different energy level in the $S^{z}=0$ subspace becomes energetically favourable which, for $a \rightarrow \infty$, decreases to zero.
With DMRG, we calculated up to five lowest eigenenergies of the system in each $S^{z}$-subspace. All higher excitation energies were found to lie, in the thermodynamical limit, infinitesimally close to the lowest excitation energy in each subspace in accordance with an analytical argument of Fáth and Sólyom [11.

\subsection{Dispersion}

As a method in real space, DMRG gives no information about the momentum of the excitations. In order to have a closer look at the dispersion curve, the low-energy spectra of smaller chains $(L \leq 14)$ were calculated by exact diagonalisation. Figures $[2$ and 3 show the dispersion of lowlying excitations at four representative points for $\sigma=-1$ (Fig. 21) and $\sigma=1$ (Fig. 3). In order to estimate the accuracy of the exact diagonalization results we have also included the DMRG data for the energy gaps. They agree very well with extrapolations from results for systems with $L \leq 14$. Interestingly, large finite-size corrections are only found in the $S^{z}=0$ subspace for small $|a|$. Later we will argue that the corresponding states can be interpreted as scattering states of two magnons. The other states found using DMRG are single magnon excitations. Their energies converge evidently faster with increasing system size.

In the following subsections we will discuss the spectra for the regions $0<|a|<a_{l}, a_{l}<|a|<2$ and $|a|>2$ separately. Figs. 2 and 3 show typical examples for each region.

\subsection{1 $0<|a|<a_{l}$}

For anisotropy parameters $0<|a|<a_{l}$ the lowest excitation is found in the subspace of $S^{z}=1$ and has momentum $k=\pi(\sigma=-1)$ or $k=0(\sigma=1)$. The lowest states in the $S^{z}=0$ and $S^{z}=2$ subspaces are degenerate. They have momentum $k=0$ and an energy which is twice the value of the gap. The corresponding states might therefore by interpretated as scattering states of two elementary magnon-type excitations with $S^{z}=1$.

\subsection{2 $a_{l}<|a|<2$}

As already mentioned in Sec. 3.2 at $a_{l} \approx 1.4$ a level crossing is observed for the second excited state. In the region $a_{l}<|a|<2$ the scattering states for small momenta still exist. However, another state with $S^{z}=0$ has now a lower energy. The momentum of the corresponding second gap, which is smaller than twice the gap in the $S^{z}=1$ subspace, for $\sigma=-1$ is now given by $k=\pi$ instead of $k=0$, and vice versa for $\sigma=1$. Later (in Sec. 4.1) we will show that this new state is well described by a single mode approximation and therefore corresponds to another single-magnon excitation. This state is result of the splitting of the Haldane triplet due to the anisotropy. 

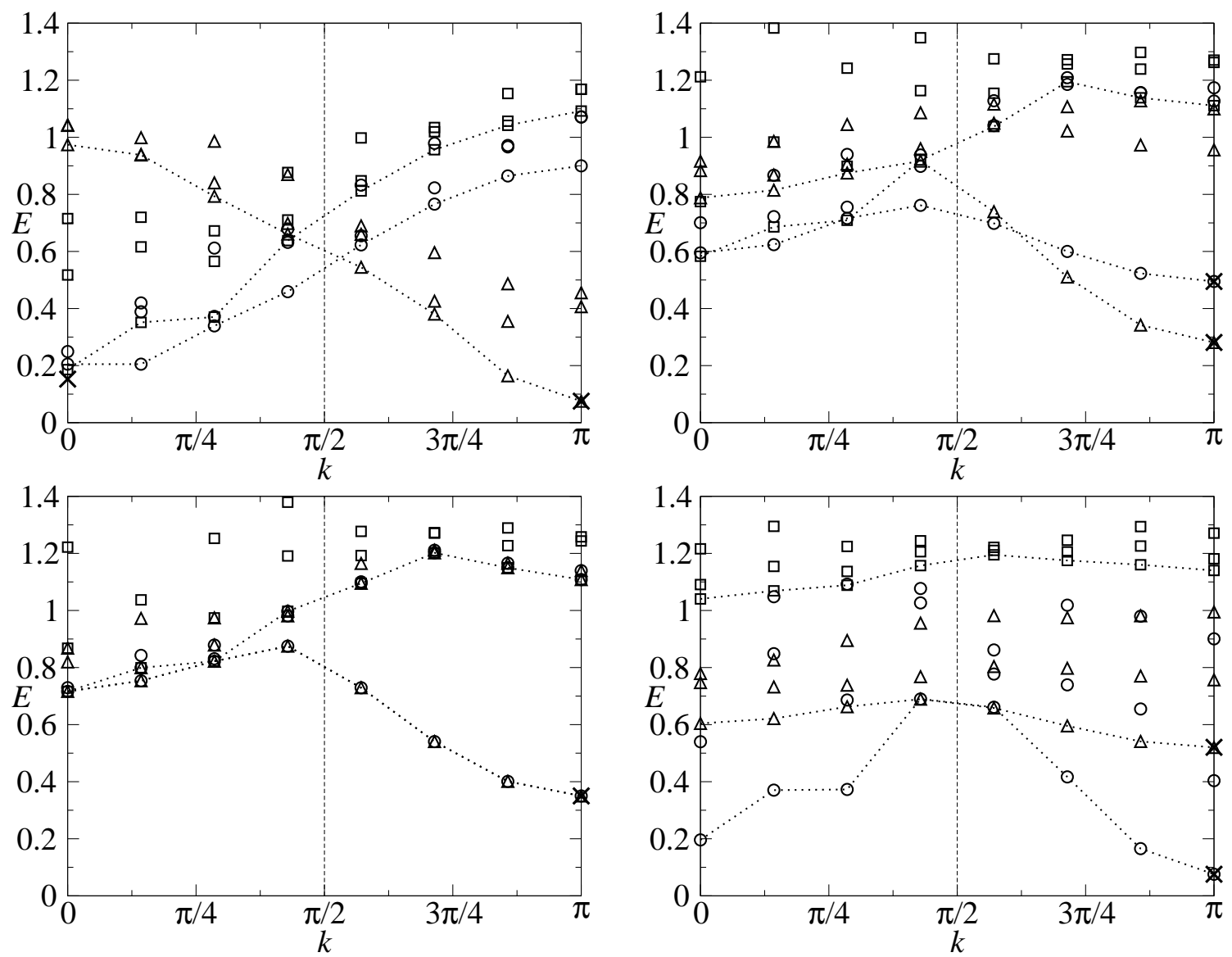

Fig. 2. Dispersion of lowest excitations in the subspaces for $S^{z}=0$ (०), $S^{z}=1 \triangle$ and $S^{z}=2$ (ם) at $a=0.5$ (top left), $a=1.5$ (top right), $a=2$ (bottom left), $a=5$ (bottom right) for $\sigma=-1$. The lowest excitation energies in each subspace are indicated by a dotted line which only serves as a guide to the eye. The data are obtained by exact diagonalisation $(L=14)$. For comparison the DMRG results $(\times)$ from Fig. 1 have also been included.

\subsection{3 $|a|=2$}

For the $|a|=2, \sigma=-1$, including the isotropic point for $a=-2$, the well-known properties of the AKLT model are reproduced (Sec. 3.1). The model's isotropy leads to a degeneracy of the $S^{z}=0,1$ excitation branches indicating the triplet character of an excitation with total spin 1 . As can easily be seen, the gap still occurs at a momentum $k=\pi$.

For $|a|=2$ and $\sigma=1$ the model is not isotropic and hence no degeneracy of the $S^{z}=0,1$ states is observed. However, the transformation $\mathcal{D}^{2}$ which changes $\sigma \rightarrow-\sigma$ also transforms the momenta $k$ of odd- $S^{z}$ states to $\pi=k$. This leads to the observed symmetry of the lowest excited states around $k=\frac{\pi}{2}$.

\subsection{4 $|a|>2$}

For $|a|>2$ the structure of the low-lying excitations changes compared to the case $|a|<2$. Now the lowest excitation is found in the $S^{z}=0$ subspace. We will later show that it again can be interpreted as a magnon. However, the energy gap still occurs for momentum $k=\pi$. Also the second excitation, which now is found in the $S^{z}=1$ subspace, takes its minimum energy at $k=\pi$ (for $\sigma=-1$ ) resp. $k=0$ (for $\sigma=1$ ). Note that for a large anisotropy $(|a|=5$ in Figs. 2] 3) all low-lying excitations (except for the first excitated state) have almost no dispersion.

\subsection{Variation of the spectral parameters}

In fig. 4 DMRG data for models with varying spectral parameters $\lambda_{0}, \lambda_{1}, \lambda_{2}$ are plotted. While increasing one of the parameters to $\lambda_{i}=2$, the others are kept constant $\left(\lambda_{j \neq i}=1\right)$. All diagrams share the qualitative appearance of the original model with $\lambda_{0}=\lambda_{1}=\lambda_{2}=1$ (fig. 1) containing the isotropic point. Again, we observe a level crossing in the $S^{z}=0$ subspace. A second level crossing occurs between levels in the subspace with $S^{z}=0$ and $S^{z}=1$ at the point, which represents the isotropic system in the original model. Note that for the increase of any $\lambda$-parameter all excitation levels are lifted compared to the original model. Yet, the different excitation branches show different scaling behaviour under the variation of the spectral parameters. 

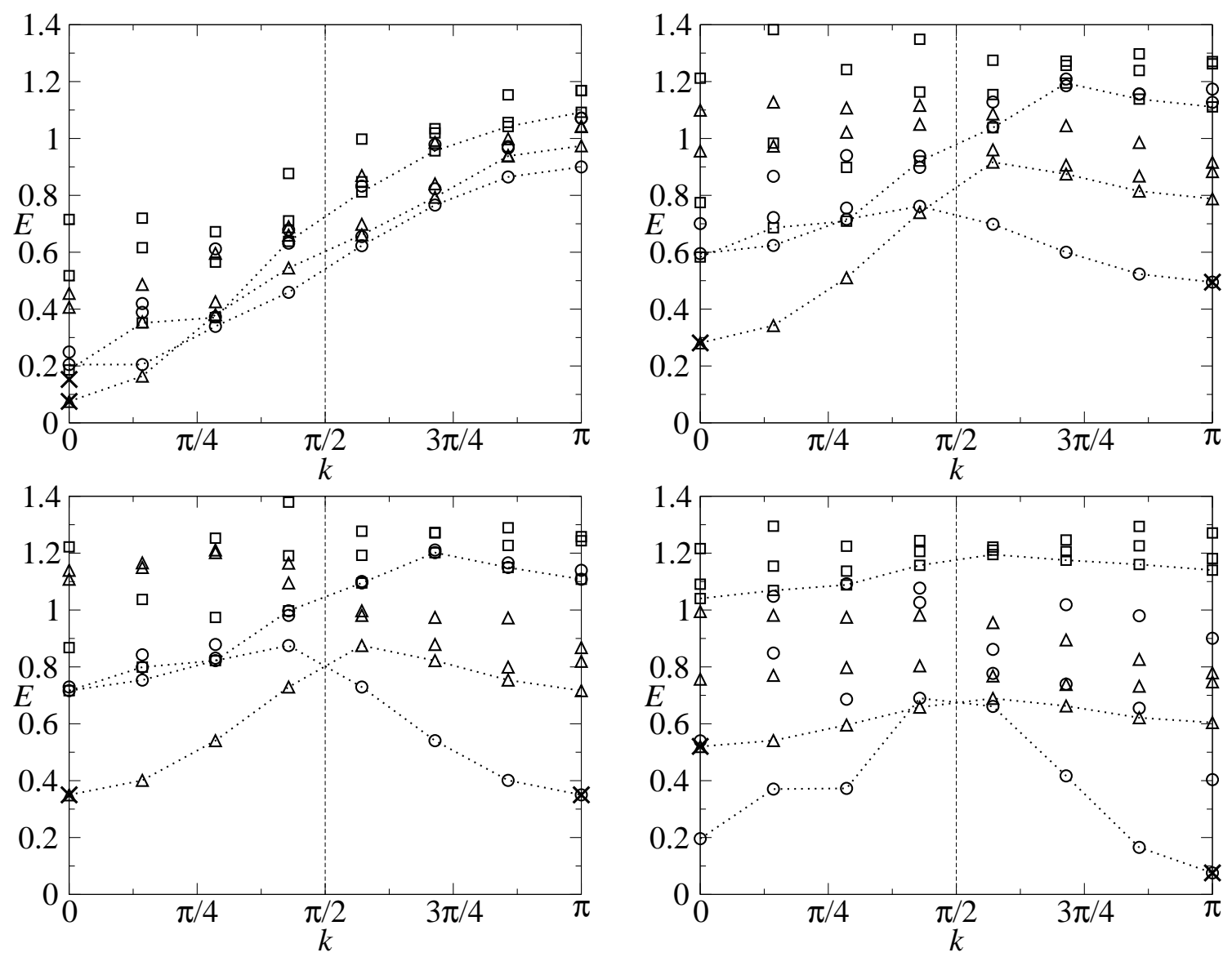

Fig. 3. Same as Fig. 2 with $\sigma=1$.

In the $S^{z}=0$ subspace with $|a|$ beyond the level crossing we find a significant $\lambda_{1}$-dependence of the decreasing excitation branch. The influence of the other spectral parameters $\lambda_{0}$ and $\lambda_{2}$ is less important. The lowest excitations in the $S^{z}=1$ and $S^{z}=2$ subspace - for small $|a|$ degenerate with the $S^{z}=0$ increasing branch - react to variations of the spectral parameters in a similar way. While $\lambda_{1}$ has strong influence in the region with small $|a|$ and while increasing $\lambda_{2}$ has greater impact in the large $|a|$ limit, there is only a weak $\lambda_{0}$-dependence. In models with small $|a|$ states with local spin configuration with no $z$-axis alignment dominate. Here, we have only a small possibility to find $(+,+)$ and $(-,-)$ adjacent spins which contribute to $\lambda_{2}$-dominated excitations. This is different in the large $|a|$ regime where spins are strongly aligned along the $z$-axis leading to the observed $\lambda_{2}$-dominance.

The similar scaling behaviour of the increasing branches in the $S^{z}=0,1,2$ subspaces suggests a common nature of these excitation levels. Particularly, it supports the picture that the increasing energy branches in the $S^{z}=0,2$ subspaces are formed by two weakly interacting magnontype excitations $\left(S^{z}=1\right)$.

\section{Single mode approximation}

In order to substantiate the interpretation of the elementary excitations as magnons, we use the single mode approximation (SMA). The SMA is a variational approach to find approximate low-lying excitations by making use of ground state correlations. Therefore, the ground state is locally perturbed similar to a spin-wave. This single mode state has a certain momentum and, since it is orthogonal to the ground state, its energy is an exact upper bound to the excitation gap. Introducing the SMA, Arovas et al. 29] gave an exact upper bound to the excitation gap of the AKLT model. In this paper, we extend their approach to the general anisotropic MPG model class in order to illustrate the spectrum of low-lying excitations.

With the locally acting perturbation operator $O_{n}$ we construct a translationally invariant state

$$
\left|\psi_{k}\right\rangle=\frac{1}{\sqrt{L}} \sum_{n=1}^{L} e^{i k n} O_{n}\left|\psi_{0}\right\rangle
$$



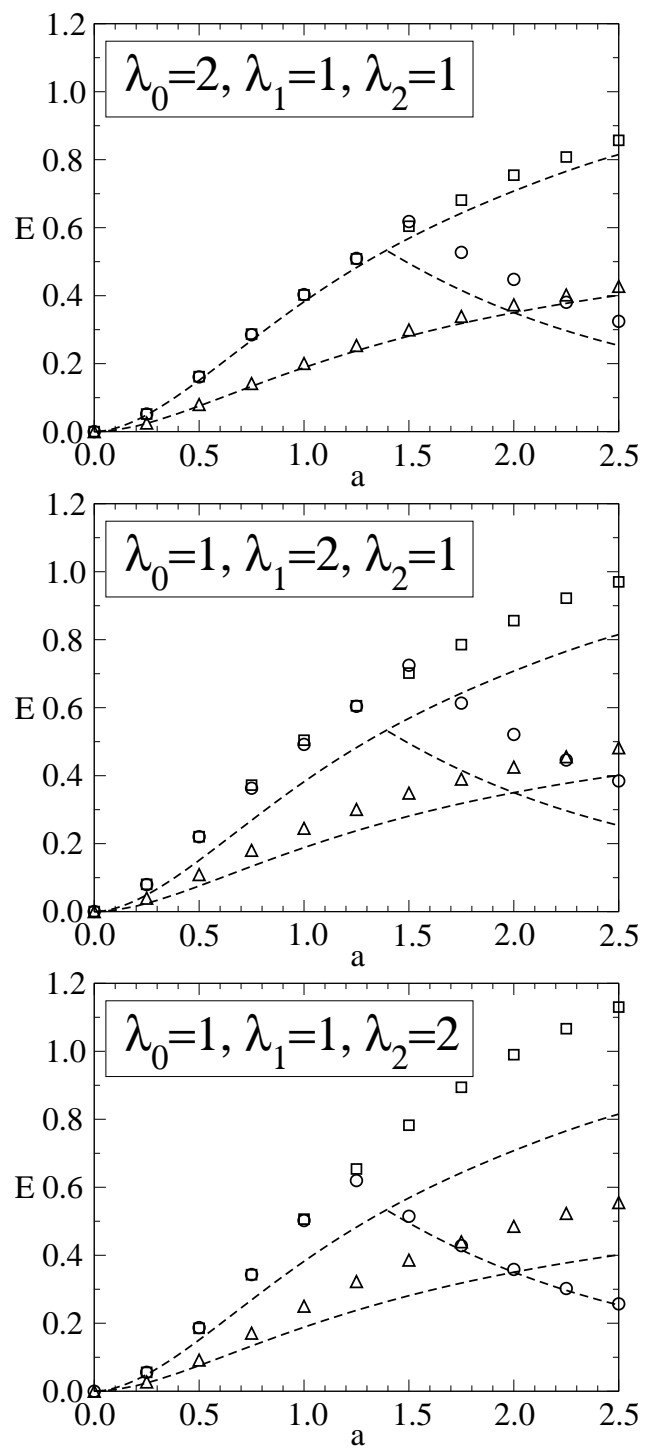

Fig. 4. Variation of the spectral parameters $\lambda_{0}, \lambda_{1}, \lambda_{2}$ (o representing the subspace $S^{z}=0, \triangle S^{z}=1$ and $\left.\square S^{z}=2\right)$. For comparison: Dashed lines represent the original model with $\lambda_{0}=\lambda_{1}=\lambda_{2}=1$. The data have been obtained from DMRG.

where $\left|\psi_{0}\right\rangle$ is the exactly known matrix product ground state (2). The energy

$$
\begin{aligned}
\omega_{k} & =\frac{\left\langle\psi_{k}|H| \psi_{k}\right\rangle}{\left\langle\psi_{k} \mid \psi_{k}\right\rangle} \\
& =\frac{\sum_{n, n^{\prime}} e^{i k\left(n-n^{\prime}\right)}\left\langle\psi_{0}\left|O_{n^{\prime}}^{\dagger} H O_{n}\right| \psi_{0}\right\rangle}{\sum_{n, n^{\prime}} e^{i k\left(n-n^{\prime}\right)}\left\langle\psi_{0}\left|O_{n^{\prime}}^{\dagger} O_{n}\right| \psi_{0}\right\rangle}
\end{aligned}
$$

of this variational state can be regarded as a quotient of expectation values of the exactly known matrix product ground state which can be calculated via the transfer matrix method [35. It is an exact upper bound to the excitation gap if the variational state is orthogonal to the ground state. Complying with conservation of the $z$-component of total spin, in the following we investigate the subspaces

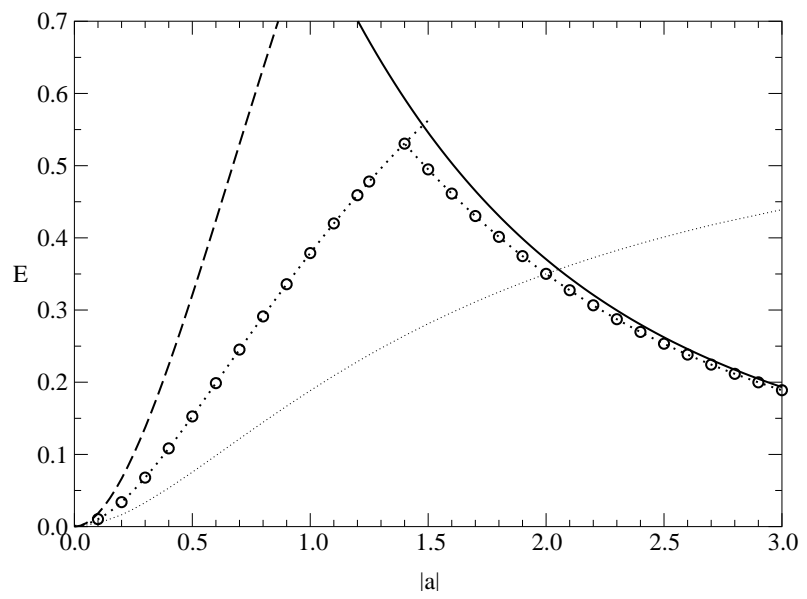

Fig. 5. Lowest excitations in the $S^{z}=0$ subspace. Numerical DMRG data (circles) and energy expectation values of the single mode approximation (dashed line $k=0$, full line $k=\pi$ ) are plotted vs. anisotropy parameter $|a|$. For comparison the thin dotted line gives the $S^{z}=1$ excitation branch (DMRG data).

with $S^{z}=1$ and $S^{z}=0$ separately. The behaviour of the gap near the boundaries of the MPG region is of special interest where it is expected to vanish.

\subsection{Subspace $S^{z}=0$ and behaviour for large $|a|$}

Considering a local application of the $S^{z}$-operator, the thus built state

$$
\left|\psi_{k}^{0}\right\rangle=\frac{1}{\sqrt{L}} \sum_{n=1}^{L} e^{i k n} S_{n}^{z}\left|\psi_{0}\right\rangle
$$

is orthogonal to the ground state $\left|\psi_{0}\right\rangle$ and has a vanishing total $S^{z}$-value. In order to find the lowest excitation with $S^{z}=0$ the most general SMA perturbation operator would include contributions of $\left(S^{z}\right)^{2}-a /(1+a)$ which is another locally orthogonal perturbation operator. However, a superposition of both possible operator in this subspace is not mandatory since one can show that variational states with only $S^{z}$ as a perturbation operator already provide the lowest SMA energy levels.

We find the variational energy

$$
\begin{aligned}
E_{k}^{0}= & \left(\frac{2|a|}{2+a^{2}} \lambda_{0}+\lambda_{1}\right) \\
& {\left[\frac{1+a^{2}}{(1+|a|)^{2}}+\frac{a^{2}-1}{(1+|a|)^{2}} \cos k\right] }
\end{aligned}
$$

as an upper bound to the lowest excitation in the $S^{z}=0$ subspace.

In the case of $|a|>2$ the excitation gap is found in the $S^{z}=0$ subspace with a momentum $\pi$. The $k=\pi$ variational energy

$$
E_{\pi}^{0}=\left(\frac{2|a|}{2+a^{2}} \lambda_{0}+\lambda_{1}\right) \frac{2}{(1+|a|)^{2}}
$$


tends to zero for $|a| \rightarrow \infty$. At the AKLT point $(|a|=2)$ the SMA variational energy $E_{\mathrm{AKLT}}=10 / 27$ of Arovas et al. 29] is reproduced. For small $|a|$ the lowest $S^{z}=0$ excited state has a momentum $k=0$.

Figure 5 shows the SMA energies in comparison to the excitation gap calculated by DMRG. For large values of $|a|$ we find the best agreement between analytical and numerical data in considering the variational state with momentum $k=\pi$. While at the point of the level crossing $(|a| \approx 1.4)$ the SMA-energy is clearly higher than the DMRG data, both analytically and numerically calculated energies become asymptotically equal in the $|a| \rightarrow \infty$ limit. For small $|a|$ the lowest excited state of this subspace has vanishing momentum. Here, the variational state with $k=0$ is not capable to replicate the numerically calculated excitation branch as suitably as in the large- $|a|$ case. This indicates a character of the small- $|a|$ excitation branch significantly different from the one-magnon picture of the excitation gap.

\subsection{Subspace $S^{z}=1$ and behaviour for small $|a|$}

In order to describe the lowest excitation in the $S^{z}=1$ subspace we use the most general local perturbation operator

$$
O_{n}^{(+)}=S_{n}^{+}\left[b+(2 b-1) S_{n}^{z}\right]
$$

comprising a variational parameter $b \in \mathbb{R}$. For $b=0$, we have $O^{(+)} \propto S^{+} S^{z}$ whereas for $b=\frac{1}{2}$ it reduces to $O^{(+)} \propto S^{+}$. The corresponding variational state

$$
\left|\psi_{k}^{+}\right\rangle=\frac{1}{\sqrt{L}} \sum_{n=1}^{L} e^{i k n} O_{n}^{(+)}\left|\psi_{0}\right\rangle,
$$

is orthogonal to the ground state and has a total $S^{z}=1$ and momentum $k$. Since in the MPG model with $\sigma=1$ we expect the excitation gap at vanishing momentum, in the following, we set $k=0$ and assume $a>0$ without loss of generality. Square of the norm

$$
\left\langle\psi^{+} \mid \psi^{+}\right\rangle=\frac{1}{1+a}\left[1+b^{2}+\frac{1}{2} a(1-b)^{2}\right]
$$

and energy expectation value

$$
\begin{aligned}
\left\langle\psi^{+}|H| \psi^{+}\right\rangle= & \frac{1}{(1+a)^{2}}\left\{\frac{1}{2} \lambda_{1} a^{2}(1-b)^{2}+2 \lambda_{2} a b^{2}\right. \\
& \left.+\frac{2 \lambda_{0} a}{2+a^{2}}[a(1-b)-b]^{2}\right\}
\end{aligned}
$$

of the variational state can be calculated via the transfer matrix method. In the following we choose the realistic spectral parameter configuration $\lambda_{0}, \lambda_{1}, \lambda_{2}=1$, including the AKLT model. Thus, we obtain the energy

$$
\begin{aligned}
E^{+} & =\frac{\left\langle\psi_{k}^{+}|H| \psi_{k}^{+}\right\rangle}{\left\langle\psi_{k}^{+} \mid \psi_{k}^{+}\right\rangle} \\
& =\frac{\frac{1}{2} a^{2}(1-b)^{2}+2 a b^{2}+\frac{2 a}{2+a^{2}}[a-b(1+a)]^{2}}{(1+a)\left[1+b^{2}+\frac{1}{2} a(1-b)^{2}\right]}
\end{aligned}
$$

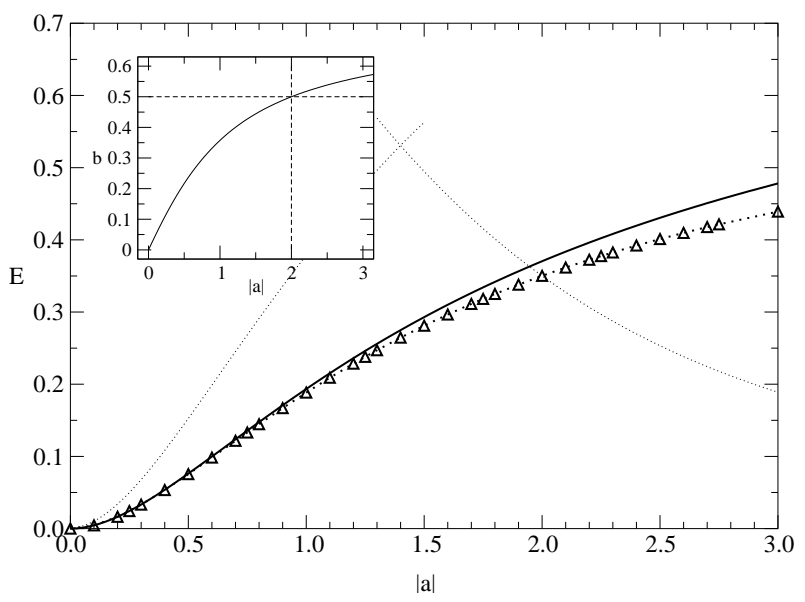

Fig. 6. Lowest excitations in the $S^{z}=1$ subspace. Numerical DMRG data $(\triangle)$ and energy expectation values of the single mode approximation (straight line) are plotted vs. anisotropy parameter $|a|$. Thin dotted lines give the $S^{z}=0$ excitation branches (DMRG data). The inset shows the minimizing variational parameter $b_{\min }$ in the same range of $|a|$.

of the variational state as a function of $a$ and $b$. Minimizing the energy of the variational state yields the condition

$$
\begin{aligned}
b_{\min }= & \frac{a^{3}+4 a^{2}+7 a+6}{a^{2}(a-2)} \\
& -\frac{\sqrt{4 a^{5}+36 a^{4}+80 a^{3}+97 a^{2}+84 a+36}}{a^{2}(a-2)}
\end{aligned}
$$

(inset of fig. [6).

In fig. [6 the energy of the variational state is depicted. A good agreement with the numerical data, particularly for small $a$, is observed. A series expansion for small $a$

$$
\begin{aligned}
b & =\frac{1}{2} a-\frac{1}{12} a^{2}+\mathrm{O}\left(a^{3}\right), \\
E^{+} & =\frac{1}{2} a^{2}-\frac{1}{2} a^{3}+\frac{1}{4} a^{4}+\mathrm{O}\left(a^{5}\right),
\end{aligned}
$$

predicts a decrease of the excitation gap of order $a^{2}$. Here, in the $a \rightarrow 0$ limit, the optimal choice of the local variational operator is $S^{+} S^{z}$. A fit of the numerical DMRG data in this region

$$
E_{\mathrm{DMRG}} \approx 0.500 a^{2}-0.497 a^{3}-0.189 a^{4}
$$

can be well reproduced by the variational calculation up to the third order in $a$. At the isotropic point $(|a|=2)$ we find $b_{\min }=\frac{1}{2}\left(S^{+}\right.$as best variational operator $)$and $E_{\mathrm{AKLT}}=10 / 27$, both in accordance with the triplet excitation picture. For large $a$ we find

$$
\begin{gathered}
b_{\min }=1-2 \sqrt{\frac{1}{a}}+\mathrm{O}\left(a^{-1}\right), \\
E^{+}=1-\sqrt{\frac{1}{a}}+\mathrm{O}\left(a^{-1}\right) .
\end{gathered}
$$




\section{Conclusion}

The energy gap and lowest excitations of the anisotropic spin-1 chain with a matrix product ground state have been subject to numerical and analytical calculations. DMRG studies showed an excitation gap for finite values of anisotropy parameter $|a|$. In the isotropic case the lowest excitation is a triplet of momentum $\pi$. Under anisotropic interactions this excitation triplet splits and gives rise to two separate excitation branches with $S^{z}=0$ and $S^{z}=1$ establishing the gap for large $|a|$ and small $|a|$, respectively.

In the large $|a|$ regime the lowest excitation can be identified as a magnon of vanishing $S^{z}$ and momentum $k=\pi$. For $|a|<2$ the excitation gap is found in the $S^{z}=1$ subspace with momentum $k=\pi(k=0)$ in case of a parameter choice $\sigma=-1(\sigma=1)$. Both numerically found excitation energies can be explained quantitatively well by the single mode approximation. From DMRG we find that the $S^{z}=2$ and the increasing $S^{z}=0$ excitation branches have twice the magnitude of the $S^{z}=1$ excitation gap. Together with results from numerical investigation of the momentum and of the scaling behaviour under variation of the spectral parameters this is strong evidence that these excitations consists of two weakly interacting $S^{z}=1$ magnons.

At $a=0$ a phase transition to a three-fold degenerate ground state with excitation continuum occurs. In its neighbourhood we observed numerically and analytically a decrease of the excitation gap with $a^{2}$.

This work has been performed within the research program of the Sonderforschungsbereich 608 supported by DFG.

\section{Appendix A}

Apart from the spin operator description, the Hamiltonian of the matrix product ground state model can also be represented by a sum of projection operators on local ground states. This spectral representation reflects the model's symmetries and helps to understand the free parameters of the MPG model.

Due to the rotational invariance in the $(x, y)$-plane we can divide the 9-dimensional Hilbert space of a twosite spin-1 system into five subspaces of conserved $S^{z}=$ $S_{1}^{z}+S_{2}^{z}$. The eigenstates of the 1-dimensional subspaces with $S^{z}=2$ are fixed. Further, parity invariance leads to discrimination of symmetric and antisymmetric states. In the 2-dimensional subspaces characterised by $S^{z}=1$ each subspace has one symmetric and one antisymmetric eigenstate. In case of $S^{z}=0$, however, after identifying one antisymmetric state, a 2-dimensional symmetric subspace leaves one degree of freedom which is described by the superposition parameter $a$. Finally, we find nine local basis states

$$
\begin{aligned}
\left|\phi_{ \pm 2}\right\rangle & =| \pm \pm\rangle, \\
\left|\phi_{ \pm 1}^{\sigma}\right\rangle & =\frac{1}{\sqrt{2}}(| \pm 0\rangle+\sigma|0 \pm\rangle), \\
\left|\phi_{0}^{-}\right\rangle & =\frac{1}{\sqrt{2}}(|+-\rangle-|-+\rangle), \\
\left|\phi_{01}^{+}\right\rangle & =\frac{1}{\sqrt{2 a^{2}+4}}(a[|+-\rangle+|-+\rangle]+2|00\rangle), \\
\left|\phi_{02}^{+}\right\rangle & =\frac{1}{\sqrt{2+a^{2}}}(|+-\rangle+|-+\rangle-a|00\rangle)
\end{aligned}
$$

with $\sigma= \pm 1$ distinguishing symmetric and antisymmetric states. Considering the projection operators $\mathcal{P}_{n}^{\sigma}:=$ $\left|\phi_{n}^{\sigma}\right\rangle\left\langle\phi_{n}^{\sigma}\right|$ onto these basis states and paying attention to the spin-flip symmetry we find a full representation of the local Hamiltonian

$$
\begin{aligned}
h_{j, j+1} & =\lambda_{2}\left(\mathcal{P}_{2}+\mathcal{P}_{-2}\right) \\
& +\lambda_{1}^{+}\left(\mathcal{P}_{1}^{+}+\mathcal{P}_{-1}^{+}\right)+\lambda_{1}^{-}\left(\mathcal{P}_{1}^{-}+\mathcal{P}_{-1}^{-}\right) \\
& +\lambda_{01}^{+} \mathcal{P}_{01}^{+}+\lambda_{02}^{+} \mathcal{P}_{02}^{+}+\lambda_{0}^{-} \mathcal{P}_{0}^{-}
\end{aligned}
$$

which depends on six spectral parameters $\lambda_{2}, \lambda_{1}^{+}, \lambda_{1}^{-}, \lambda_{01}^{+}$, $\lambda_{02}^{+}, \lambda_{0}^{-}$and the superposition parameter $a$. The spin-chain has a MPG if $\lambda_{2}, \lambda_{1}^{-\sigma}, \lambda_{02}^{+}>0$ and $\lambda_{1}^{\sigma}, \lambda_{01}^{+}, \lambda_{0}^{-}=0$ leading to a local MPG Hamiltonian

$$
h_{j, j+1}=\lambda_{2}\left(\mathcal{P}_{2}+\mathcal{P}_{-2}\right)+\lambda_{1}\left(\mathcal{P}_{1}^{-\sigma}+\mathcal{P}_{-1}^{-\sigma}\right)+\lambda_{0} \mathcal{P}_{02}^{+}
$$

with $\lambda_{1}=\lambda_{1}^{\sigma}$ and $\lambda_{0}=\lambda_{02}^{+}$. Spin and spectral parameters of the MPG model class are mapped onto each other by

$$
\begin{aligned}
& J_{1}=-\frac{1}{2} \sigma \lambda_{1}, \quad \Delta=-\sigma \frac{\lambda_{2}}{\lambda_{1}}, \quad J_{2}=\frac{1}{2+a^{2}} \lambda_{0}, \\
& J_{3}=\frac{a^{2}}{2+a^{2}} \lambda_{0}-\lambda_{1}+\frac{1}{2} \lambda_{2}, \\
& J_{4}=\frac{a}{2+a^{2}} \lambda_{0}-\frac{1}{2} \sigma \lambda_{1}, \\
& D=\frac{1-a^{2}}{2+a^{2}} \lambda_{0}+\frac{1}{2} \lambda_{1}, \quad c=\frac{a^{2}-2}{a^{2}+2} \lambda_{0} .
\end{aligned}
$$

\section{References}

1. I. Bose, Low-dimensional quantum spin systems, condmat/0011262, 2000.

2. D. Mermin and H. Wagner, Phys. Rev. Lett. 17, 1133 (1966).

3. F. Haldane, Phys. Lett. 93A, 464 (1983).

4. H. Bethe, Z. Phys. 71, 205 (1931).

5. S. R. White and D. A. Huse, Phys. Rev. B 48, 3844 (1993).

6. H. Aschauer and U. Schollwöck, Phys. Rev. B 58, 359 (1998).

7. J. Sólyom, Phys. Rev. B 36, 8642 (1987).

8. N. Papanicolaou, Nucl. Phys. B 305, 367 (1988).

9. I. Affleck, J. Phys. CM 1, 3047 (1989).

10. G. Fáth and J. Sólyom, Phys. Rev. B 44, 11836 (1991).

11. G. Fáth and J. Sólyom, Phys. Rev. B 47, 872 (1993).

12. G. Fáth and J. Sólyom, J. Phys. CM 5, 8983 (1993). 
13. I. Affleck, Rev. Math. Phys. 6, 887 (1994).

14. G. Fáth and J. Sólyom, Phys. Rev. B 51, 3620 (1995).

15. T. I. K. Tanaka, A. Tanaka, J. Phys. A 34, 8767 (2001).

16. I. Affleck, T. Kennedy, E. H. Lieb, and T. Tasaki, Phys. Rev. Lett. 59, 799 (1987).

17. B. Sutherland, Phys. Rev. B 12, 3795 (1975).

18. G. V. Uimin, JETP Lett. 12, 225 (1976).

19. A. Klümper, Europhys. Lett. 9, 815 (1989).

20. L. A. Takhtajan, Phys. Lett. 87A, 479 (1982).

21. H. M. Babujian, Phys. Lett. 90A, 479 (1982).

22. W. J. L. Buyers et al., Phys. Rev. Lett. 56, 371 (1986).

23. H. Mutka, J. L. Soubeyroux, G. Bourleaux, and P. Colombet, Phys. Rev. B 39, 4820 (1989).

24. J. P. Renard et al., J. Appl. Phys. 63, 3538 (1988).

25. J. Darriet and L. P. Regnault, Solid State Commun. 86, 409 (1993).

26. J. F. DiTusa et al., Physica B 194-196, 181 (1994).

27. F. Mila and F. C. Zhang, Eur. Phys. J. B 16, 7 (2000).

28. A. Klümper, A. Schadschneider, and J. Zittartz, Europhys. Lett. 24, 293 (1993).

29. D. P. Arovas, A. Auerbach, and F. D. M. Haldane, Phys. Rev. Lett. 60, 531 (1988).

30. I. Affleck, T. Kennedy, E. H. Lieb, and T. Tasaki, Commun. Math. Phys. 115, 477 (1988).

31. S. R. White, Phys. Rev. Lett. 69, 2863 (1992).

32. S. R. White, Phys. Rev. B 48, 10345 (1993).

33. I. Peschel, W. Wang, M. Kaulke, and K. Hallberg, editors, Density Matrix Renormalisation. A New Numerical Method in Physics, volume 528 of Lecture Notes in Physics, Springer, Berlin, 1998.

34. W.-D. Freitag, Anregungen der Spin 1 Valence-BondSolid-Kette, PhD thesis, Institut für Theoretische Physik, Universität zu Köln, 1993.

35. A. Klümper, A. Schadschneider, and J. Zittartz, Z. Phys. B 87, 281 (1992). 